\title{
Correction of estimation bias of predictive equations of energy expenditure based on wrist/waist-mounted accelerometers
}

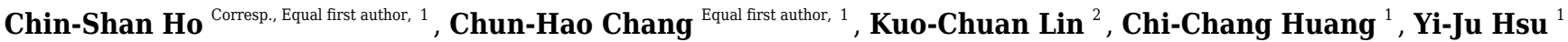 \\ ${ }^{1}$ Graduate Institute of Sports Science, National Taiwan Sport University, Taoyuan, Taiwan \\ 2 Office of Physical Education, Chung Yuan Christian University, Taoyuan, Taiwan \\ Corresponding Author: Chin-Shan Ho \\ Email address: kilmur33@gmail.com
}

Background: Using wearable inertial sensors to accurately estimate energy expenditure (EE) during an athletic training process is important. Due to the characteristics of inertial sensors, however, the positions in which they are worn can produce signals of different natures. To understand and solve this issue, this study used the heart rate reserve (HRR) as a compensation factor to modify the traditional empirical equation of the accelerometer EE sensor and examine the possibility of improving the estimation of energy expenditure for sensors worn in different positions.

Methods: Indirect calorimetry was used as the criterion measure (CM) to measure the EE of 90 healthy adults on a treadmill (5 speeds: $4.8,6.4,8.0,9.7$, and $11.3 \mathrm{~km} / \mathrm{h}$ ). The measurement was simultaneously performed with the ActiGraph GT9X-Link (placed on the wrist and waist) with the Polar H10 Heart Rate Monitor.

Results: At the same exercise intensity, the EE measurements of the GT9X on the wrist and waist had significant differences from those of the $\mathrm{CM}(p<0.05)$. By using multiple regression analysis - utilizing values from vector magnitudes (VM), body weight (BW) and HRR parameters -accuracy of EE estimation was greatly improved compared to traditional equation. Modified models explained a greater proportion of variance $\left(R^{2}\right)$ (wrist: 0.802; waist: 0.805 ) and demonstrated a good ICC (wrist: 0.863, waist: 0.889) compared to Freedson's VM3 Combination equation ( $R^{2}$ : wrist: 0.384 , waist: 0.783 ; ICC: wrist: 0.073 , waist: 0.868).

Conclusions: The EE estimation equation combining the VM of accelerometer measurements, BW and HRR greatly enhanced the accuracy of EE estimation based on data from accelerometers worn in different positions, particularly from those on the wrist. 


\section{Correction of estimation bias of predictive equations of} 2 energy expenditure based on wrist/waist-mounted

\section{3 accelerometers}

6 Chin-Shan $\mathrm{Ho}^{1}$, Chun-Hao Chang ${ }^{1}$, Kuo-Chuan Lin ${ }^{2}$, Chi-Chang Huang ${ }^{1}$, Yi-Ju Hsu ${ }^{1}$ 7

$8{ }^{1}$ Graduate Institute of Sports Science, National Taiwan Sport University, Taoyuan, Taiwan

92 Office of Physical Education, Chung Yuan Christian University, Taoyuan, Taiwan 10

11 Corresponding Author:

12 Chin-Shan $\mathrm{Ho}^{1}$

13 No. 250, Wenhua 1st Rd., Guishan District, Taoyuan City, 33301, Taiwan 


\section{Abstract}

18 Background: Using wearable inertial sensors to accurately estimate energy expenditure (EE) 19 during an athletic training process is important. Due to the characteristics of inertial sensors, 20 however, the positions in which they are worn can produce signals of different natures. To 21 understand and solve this issue, this study used the heart rate reserve (HRR) as a compensation 22 factor to modify the traditional empirical equation of the accelerometer EE sensor and examine 23 the possibility of improving the estimation of energy expenditure for sensors worn in different 24 positions.

25 Methods: Indirect calorimetry was used as the criterion measure (CM) to measure the EE of 90 26 healthy adults on a treadmill (5 speeds: $4.8,6.4,8.0,9.7$, and $11.3 \mathrm{~km} / \mathrm{h}$ ). The measurement was 27 simultaneously performed with the ActiGraph GT9X-Link (placed on the wrist and waist) with 28 the Polar H10 Heart Rate Monitor.

29 Results: At the same exercise intensity, the EE measurements of the GT9X on the wrist and waist had significant differences from those of the $\mathrm{CM}(p<0.05)$. By using multiple regression analysis 31 - utilizing values from vector magnitudes (VM), body weight (BW) and HRR parameters accuracy of EE estimation was greatly improved compared to traditional equation. Modified models explained a greater proportion of variance $\left(\mathrm{R}^{2}\right)$ (wrist: 0.802 ; waist: 0.805 ) and 34 demonstrated a good ICC (wrist: 0.863, waist: 0.889) compared to Freedson's VM3 Combination 35 equation ( $\mathrm{R}^{2}$ : wrist: 0.384 , waist: 0.783 ; ICC: wrist: 0.073 , waist: 0.868$)$.

36 Conclusions: The EE estimation equation combining the VM of accelerometer measurements, 37 BW and HRR greatly enhanced the accuracy of EE estimation based on data from accelerometers 38 worn in different positions, particularly from those on the wrist. 


\section{Introduction}

42 Regular exercise is a cost-effective and efficient method of promoting and maintaining physical

43 health and performance. Additionally, high levels of physical activity are closely related to the decreased risk and mortality rates of chronic diseases (Delisle et al., 2010; Kokkinos \& Myers 2010; Lawrence et al., 2014; González et al. 2017). In recent years, the accelerated development of smart products have made modern life more dependent on technology. This has resulted in a faster pace of life and significantly increased the pressures of work and life, leaving less time for exercise and rest. An increase in obesity rates across developing/developed countries has been associated with this technological era (Żukiewicz-Sobczak et al., 2014; Poobalan \& Aucott, 2016). Obesity has a detrimental impact on human health; it is now widely recognized that regular exercise and increased physical fitness is important to maintain one's health. Both the American Heart Association (AHA) and the American College of Sports Medicine (ACSM) have developed physical activity guidelines with recommendations for sufficient volumes and intensities of energy expenditure (EE). It is also recommended that treatment plans within the healthcare system include regular assessments of physical activity (Garber et al. 2011; ACSM, 2019; AHA 2019).

When assessing body weight management, inaccurate EE measurement can result in insufficient or excessive intake of nutrients, leading to a decreased amount of muscle tissues or the increased development of adipose tissues, respectively. This may also have significant implications for a person's health; the body's cardiovascular system, endocrine system, musculoskeletal system, thermoregulation, growth, and physical development can all be impacted (Arieli \& Constantini, 2012; Burrows et al., 2016). Typical measurement methods of quantifying the EE and intensity of physical activity are doubly labelled water (DLW) and indirect calorimetry (Gao et al., 2012; Brage et al., 2015; Beltrame et al., 2016; Lee et al., 2016; Westerterp, 2017). Nevertheless, because of the costs and technical expertise required for these techniques, these methods are not commonly accessible in community settings. Due to continuous improvement of accelerometer-based wearable sensors, it is now easier to estimate EE and exercise intensity. As wearable sensors are easy-to-use and economical, and because they can continuously measure physical activity for a number of days (Shih, Ho \& Shiang, 2014; Montoye et al., 2015; Menai et al., 2017; Xu et al., 2018), they have become a "popular" accessory for both general population and professional athletes who are undertaking exercise training.

The development of EE estimation equations has largely relied on treadmill exercise tests and daily activity tests as the experimental parameters, where the vector magnitude (VM) activity counts are used for the subsequent calculations (Crouter, Clowers \& Bassett, 2006; Lyden, et al., 2011; Sirichana et al., 2017). The accelerometer-based wearable inertial sensors on the market are usually placed on the wrist or waist to provide referential EE estimates for different types of activities. Although some researchers reported that the wrist-mounted device can be used to 
demonstrated that waist-mounted devices worn closer to the body centre of mass can provide EE estimates that are more accurate than those of wrist-mounted devices (Hildebrand et al., 2014; Tudor-Locke, Barreira \& Schuna, 2015; Chomistek et al., 2017). It is also important to note that accelerometers can cause overestimation or underestimation of EE based on different exercise types or intensities (i.e. cycling, uphill exercise, etc) (Schneller et al. 2015; Tarp, Andersen \& Ostergaard, 2015; Yang et al., 2018; Kuo et al., 2018; Chang et al. 2019).

The smart watch is currently a popular device worldwide and is widely discussed as a means to monitor EE. In recent years, the technology of combining the smart watch with the optical heart rate sensor has matured, improving HR (heart rate) monitoring. Due to the limitations of accelerometers, wearing the device on different parts of the body can produce acceleration signals of different natures and create bias in the data. Therefore, some researchers have used HR to modify customized exercise volumes in order to correct the measurement error caused by different wearing positions, to better estimate $\mathrm{EE}$, and to obtain corrected parameters for modifying the $\mathrm{EE}$ estimation equation (Domene \& Easton 2014; Oppert et al., 2016; Kuo et al., 2018). Our previous study found that adding HRR parameters to the estimation equation could increase the accuracy of EE estimation more than HR parameters (Chang et al. 2019). Therefore, we hypothesised that the accuracy of EE estimation from wrist- and waist-worn accelerometers may be improved by applying a HRR correction. The purpose of this research was to modify the traditional EE estimation equation (Freedson's VM3 Combination equation, 2011), which is suitable for devices worn on different parts of the body.

\section{Materials \& Methods}

\section{Study design}

In this study, indirect calorimeter and accelerometer monitoring devices were used to measure the EE of healthy adults, and an accelerometer-based HR monitoring device was simultaneously used to measure their HR during the exercise tests. The HRR parameters were used to modify the EE estimation equation to make it applicable to both wrist- and waist-mounted devices. The study procedures were approved by the Institutional Review Board of Fu Jen Catholic University (Approval number: C106056). All participants were informed of the study procedures and provided written informed consent prior to the testing.

\section{Subjects}

Ninety healthy adults voluntarily participated in this study. People who had exercise contraindications, were taking drugs that could change the metabolic rate, or had cardiovascular abnormalities were excluded from this research, as such circumstances could prevent them from completing the test safely. Subjects were asked to wear experimental devices and complete a one- 
115 hour test (including the time spent mounting the device on the body) in a laboratory at a room 116 temperature of $23^{\circ} \mathrm{C}$ (on average). If a subject failed to complete the test (e.g., failed to maintain 117 a specified speed or withdrew before completion), the test was terminated immediately, and the 118 subject's personal information and data were removed from the dataset. The subjects' 119 anthropometric data are listed in Table 1.

120

121

122

123

124

125

126

127

128

129

130

131

132

133

134

135

136

137

138

139

140

141

142

143

144

145

146

147

148

149

150

151

\section{Measurement of EE by indirect calorimeter and accelerometer methods}

Regarding the indirect calorimeter method, the Cardiopulmonary Exercise Testing System (Vmax Encore 29 System, VIASYS Healthcare Inc, Yorba Linda, CA) was adopted to conduct the metabolic criterion measure (CM). The device was warmed up for at least 15 minutes and then calibrated before each of the tests. Test subjects wore a Hans-Rudolph mask that covered the nose and mouth. A sampling line and flow sensor connected to the mask were used to measure the volume and composition of inspired and expired air.

Regarding the accelerometer method, the ActiGraph GT9X-Link (ActiGraph Corporation, Pensacola, FL, USA) was used. This device measures accelerations across 3 -axis and is small (3.5 $\mathrm{cm} \times 3.5 \mathrm{~cm} \times 1 \mathrm{~cm})$ and lightweight $(\sim 14 \mathrm{~g})$. Before each test, the ActiLife6 software (version 6.12.1, Cary, NC, USA) was used to initialize the GT9X-Link and set the compatible chestmounted Polar H10 Heart Rate Monitor (Polar Electro Oy, Finland). The sampling frequency of the monitoring device was $30 \mathrm{~Hz}$, and the activity count and HR data were collected in 10 -second epochs. The user manual of the ActiGraph recommends that the GT9X-Link was affixed to the wrist of the subject's non-dominant hand and the right hip on the midaxillary line (one on wrist, the other on waist) by an adjustable soft elastic belt. The test subjects were arranged to take one test at a time, and five treadmill speeds walking/running tests were conducted in a randomized order. During the process, $\mathrm{VO}_{2}$ (oxygen uptake) by indirect calorimetry, $\mathrm{HR}$ and accelerometer counts for all tests were simultaneously and continuously recorded. The times of the Vmax and GT9X-Link were synchronously initiated according to the clock of the ActiGraph.

\section{Treadmill test}

Subjects were requested to perform walking/running tests at speeds of 4.8, 6.4, 8.0, 9.7, and 11.3 $\mathrm{km} / \mathrm{h}$. Each speed test was at least 3 minutes, and all tests were separated by two-minute breaks (test method adapted from Tudor-Locke, Barreira \& Schuna, 2015). If the subject failed to complete the test safely (e.g., was unable to maintain the treadmill speed) or the subject's heart rate exceeded the safety limit (220 minus the participant's age) during the exercise test, the test was terminated and any relevant data was deleted from the analysis.

\section{Data analysis}


152 All ninety of the test subjects completed the exercise tests safely, therefore all Vmax, HR and 153 GT9X-Link data were outputted into Microsoft Excel. The Vmax and HR data were used to 154 calculate the parameters of the 10s-by-10s time series and were synchronized with the ActiGraph 155 GT9X-Link accelerometer data. The data processing followed the approach of Lyden et al. (2011) 156 whereby the first 120 seconds of each speed were removed to ensure that the participant achieves stability in movement under the exercise intensity. The $\mathrm{VO}_{2}$ and carbon dioxide output $\left(\mathrm{VCO}_{2}\right)$ were calculated to determine $\mathrm{EE}$ by Weir's equation: $\mathrm{EE}\left(\mathrm{Kcals} \cdot \mathrm{min}^{-1}\right)=3.491\left(\mathrm{VO}_{2}\right.$ in $\left.\mathrm{L} / \mathrm{min}\right)+$ $1.106\left(\mathrm{VCO}_{2}\right.$ in $\left.\mathrm{L} / \mathrm{min}\right)($ Weir, 1949). The GT9X-Link data were analyzed by the GT9X-Link, and the EE was calculated based on the Freedson's VM3 Combination equation (2011). The equation is as follows: Kcals $\cdot \mathrm{min}^{-1}=0.001064 \mathrm{VM}+0.087512 \mathrm{BW}-5.500229$. All of the EE values were divided by the weight for standardization and are presented in $\mathrm{kcal} \cdot \mathrm{min}^{-1} \cdot \mathrm{kgw}^{-1}$. The equation HRR $=\mathrm{HR}_{\max }-\mathrm{HR}_{\text {rest }}$ refers to the difference of $\mathrm{HR}_{\max }$ and $\mathrm{HR}_{\text {rest }}$ for each phase of the test. $\mathrm{HR}_{\text {rest }}$ is the pre-test measure of resting HR.

167

168

169

170

171

172

173

174

175

176

177

178

179

180

181

182

183

184

185

186

187

188

189

\section{Statistical analysis}

The statistical software IBM SPSS Statistics version 20 (IBM Corp., New York, NY, USA) was used for statistical analysis. All data were summarized as means \pm standard deviations. In order to understand how device placements affected EE estimation across different speeds, and the difference between criterion measured EE (CMEE) and device measured EE, one-way ANOVA with Games-Howell post hoc test, Cohen's d effect size (ES) and Mean Absolute Percentage Error (MAPE) were all calculated accordingly. Linear regression was used to modify the EE estimation model with the variables of VM activity counts, body weight and HRR. Validity and reliability of EE estimation was further evaluated using the criterion validity, namely the Pearson coefficient of determination (r) and Intraclass correlation coefficient (ICC), respectively. The significance level was set to $\alpha=0.05$.

\section{Results}

The CMEE and GT9X EE (wrist and waist) data of the treadmill tests and the ES, MAPE and ICC of these two systems' measurement results are summarized in Table 2. The ANOVA test results of the CMEE and GT9X EE accelerometer data of the wrist- and waist-mounted devices indicated that in all speed test results, CMEE and GT9X EE data had significant differences $(p<0.001)$, and CMEE was higher than GT9X EE $(p<0.001$, t-test with Games-Howell post hoc). The EE variation, estimated by the wrist-mounted device, was less than the CMEE and the waist-mounted device, resulting in a non-homogeneous condition. Therefore, the Game-Howell post hoc was used for verification. As shown in that Table 2, the GT9X EE (wrist and waist) values were lower than those of the CMEE, the wrist data had a high degree of difference in effect size (ES: 1.58 to 11.54) and MAPE (21.4 to 63.9\%), and there was no significant correlation (r) in each individual speed as well as there being poor validity $(\mathrm{ICC}=0.073)$. The waist data indicated a lower degree of 
190

191

192

193

194

195

196

197

198

199

200

201

202

203

204

205

206

207

208

209

210

211

212

213

214

215

216

217

218

219

220

221

222

223

224

225

226

227

228

difference in effect size (ES: 0.36 to 1.08 ) and MAPE (4.7 to 10.4\%). A significant correlation was achieved at speeds $4.80,6.42$, and $9.66 \mathrm{~km} / \mathrm{h}(\mathrm{r}=0.529,0.428$, and 0.210 , respectively) as well as good validity $(\mathrm{ICC}=0.868)$.

Table 3 presents the results of multiple regression analysis of the EE estimation model composed of VM activity counts, body weight and HRR. The two modified models had high coefficients of determination $\left(\mathrm{R}^{2}=0.802\right.$ to 0.805$)$ and a small standard error of estimate (SEE). The criterion validity ( $\mathrm{r}$ and $\mathrm{ICC}$ ) results of CMEE and EE of different device positions estimated from the Freedson's VM3 Combination equation (2011) and modified models are listed in Table 4. From that table, it can be seen that the $\mathrm{r}$ and ICC values of the modified models ( $\mathrm{r}$ : wrist $=0.895$, waist $=0.897$, very strong correlation; ICC: wrist $=0.863$, waist $=0.889$, good ICC) were greater than those of Freedson's VM3 Combination equation (2011) (r: wrist $=0.620$, waist $=0.885$, strong to very strong correlation; ICC: wrist $=0.073$, waist $=0.868$, poor to good ICC).

\section{Discussion}

This study examined the effects of device measurement position on accelerometer outputs in estimating EE, and explored the possibility of modifying the estimation equation used previously. The main finding of this study was that the modified equation with HRR parameters significantly improved the accuracy of the estimated EE of the wrist-mounted device, while the waist-mounted device offers no significant improvement. From the results, it was clear that, when tested at different speeds, criterion measurement provided higher EE values than the wrist- and waistmounted devices $(p<0.05)$, except for waist EE at $4.8 \mathrm{~km} / \mathrm{h}(p>0.05)$. This study adopted Freedson's VM3 Combination equation (2011), and the measurement results indicated that, although this equation underestimated the EE of devices worn on the waist, it still provided a good forecast (MAPE: 4.7 to $10.4 \%, \mathrm{ICC}=0.868$ ). In addition, the EE estimated value bias of the wrist position was the greatest, and it was observed that as the speed increased, the GT9X EE prediction value of wrist did not increase significantly with speed and had poor prediction ability (MAPE: 21.4 to $63.9 \%$, ICC $=0.073$ ). If wrist-mounted monitoring is adopted, it will affect not only EE estimates, but it potentially could also affect health and/or fitness outcomes for the user. According to our previous study, HRR parameters can be used to calibrate differences in physical fitness and standardize an individual's physical fitness level (Chang et al. 2019). In this study, HRR parameters were also added to the traditional estimation equation (Freedson's VM3 Combination equation, 2011). This addition indeed improved the validity of the EE estimates of wrist- and waist-mounted devices. The ICC of modified models in the wrist position was 0.863 , and that of the waist position was 0.889 . This strongly suggests that the combination of HRR, accelerometer outputs and body weight can increase the accuracy of EE estimates for devices worn on different parts of the body.

Accelerometer-based EE sensors carry out calculations using VM activity counts produced during physical activity. A limitation of the accelerometers in the sensors is that they can create different vibration signals due to differences in the positions in which the devices are worn. In

Peer) reviewing PDF | (2019:06:38267:2:0:NEW 28 Sep 2019) 
229

230

231

232

233

234

235

236

237

238

239

240

241

242

243

244

245

246

247

248

249

250

251

252

253

254

255

256

257

258

259

260

261

262

263

264

265

266

267

other words, with the same exercise intensity, VM activity counts can increase or decrease due to differences in device positions, leading to bias in EE estimates. In the current study, it was found that the speed measurement results of the wrist position were underestimated $(p<0.05)$, that there was a large ES (wrist: 1.58 to 11.54), and that the bias could increase following increases in speed. The accelerometer-based EE estimation models were built on a database of healthy adults in which the subjects wore accelerometers on the waist for exercise tests (Crouter, Clowers \& Bassett, 2006; Lyden et al., 2011), therefore, such models are more suitable for estimating the EE measured from the waist-mounted devices. Despite a significant difference between the GT9X EE data of the waist-mounted device and the criterion measurement in all speed tests $(p<0.05)$, it still provided a lower degree of difference in the ES (waist: 0.36 to 1.08$)$, good validity $(r=0.885)$ and reliability $(\mathrm{ICC}=0.868)$. Therefore, when ignoring the difference in vibration signals produced by devices worn in different positions, this kind of non-standard measurement methods (i.e. physical activity sensors) may create different levels of bias.

As the waist is located closer to the center of body mass than wrist, it is the ideal position for wearing an accelerometer device for measuring a subject's overall physical activity. Recent researchers have also confirmed that compared to wrist-mounted accelerometer-based devices, waist-mounted devices can provide more valid (and/or reliable) estimates of EE (Hildebrand et al., 2014; Tudor-Locke et al., 2015; Chomistek et al., 2017). Nevertheless, smart watches and wrist-mounted sensors have become a trend in recent years. Therefore, it is necessary to increase the accuracy of wrist-mounted EE monitoring devices. Furthermore, it is important to note that EE (metabolism) is a complicated physiological mechanism that cannot be accurately measured using only the accelerometer. In addition, physical activity may be misclassified if accelerometers are placed in different positions which then produce signals of different strengths. Based on the results of the current investigation, when the EE estimation equation is modified it is recommended to take physiological parameters into consideration. All physical activity involves muscle contraction, which has an energy cost. When the skeletal muscle is moving, the autonomic nervous system will regulate the functions of the cardiovascular system to satisfy the energy and metabolism demands. Moreover, the control of the autonomic nervous system over HR is a dynamic regulation process (Fisher, Young \& Fadel, 2015; Dong, 2016; Chen et al., 2017). Much previous research has also indicated a close linear correlation between $\mathrm{HR}$ and $\mathrm{VO}_{2}$ which is why changes in HR can be used to evaluate exercise intensity (Strath et al. 2001; Bouchard \& Trudeau 2008; Villars et al. 2012; Domene \& Easton, 2014; Rousset et al. 2015; Colosio et al., 2018).

As a result, many researchers have suggested that accelerometer-based EE sensors can be used in conjunction with HR monitoring devices to increase the accuracy of EE estimates. Domene \& Easton (2014) indicated that Latin dance has relatively more gestures than other forms and discussed the validity of combining wrist- and waist-mounted triaxial accelerometers with heart rate measuring devices, and they evaluated the physiological and activity parameters of Latin gestures. In their study, they recruited 22 dancers to estimate their EE using accelerometer and HR data, and they modified the estimation equation based on the EEs measured using indirect

Peer) reviewing PDF | (2019:06:38267:2:0:NEW 28 Sep 2019) 
268

269

270

271

272

273

274

275

276

277

278

279

280

281

282

283

284

285

286

287

288

289

290

291

292

293

294

295

296

297

298

299

300

301

302

303

304

305

306

calorimetry. Their results determined that wrist-mounted accelerometers combined with HR data provide accuracy comparable to criterion measurement (indirect calorimetry) results. Kuo et al. (2017) discussed the possibility of improving the EE of uphill hiking using an EE estimation model established based on accelerometer and HR parameters. Their results indicated that the estimation equation could also increase the accuracy of estimating the EE of uphill hiking when the parameters of the accelerometer and HR changes were included. Chang et al. (2019) also studied the accuracy of EE estimates in an uphill walking or running activity using accelerometers, where HR and HRR were used to modify the traditional empirical equation of accelerometers as compensation factors. They found that, compared with HR, HRR provides better estimates when it is combined with the parameters of the accelerometer. The results of this study are consistent with those of previous research. As it has been proven that the initial value of HR can be variable due to an individual's physical fitness or psychological factors and can affect the accuracy of EE estimation, HRR can therefore be used as a compensation factor to correct for the user's physical fitness and improve the estimate of exercise intensity, making the estimate closer to the actual value. The mean error rates of GT9X-EE and CMEE derived from the modified models were 8.0\% for the wrist and $5.2 \%$ for the waist. The difference shows that the EE estimate of wrist-mounted devices greatly reduced the mean error rate (from $47.4 \%$ to $8.0 \%$ ) and increased the validity (r: 0.620 to 0.895 ), and reliability (ICC: 0.073 to 0.863 ).

It is important to accurately measure or estimate EE during physical activity or exercise. A key factor in enhancing training outcomes is ensuring individuals have an adequate energy balance, that is, energy intake and EE are matched. Inaccurate EE estimates can result in insufficient or excessive intake of nutrients, leading to decreases in muscle tissues or increases in adipose tissues, respectively. Such changes can not only affect an individual's athletic performance but also have a negative impact on their health. Moreover, HRR, which is the difference between maximal heart rate and resting heart rate, can correct for excessive resting heart rates caused by differences in physical fitness and be used for estimating EE or exercise intensity. This study and our previous study strongly indicate that the prediction equation contains HRR parameters, the main difference between Freedson's VM3 Combination equation (2011) (traditional EE prediction equation) and the modified model is the HRR factor, which has better EE prediction ability than the traditional EE prediction equation. Including HRR not only improved the reliability and effectiveness of estimates but also increased the accuracy of the estimation model, particularly that of devices worn on the wrist.

\section{Conclusions}

In conclusion, the Freedson's VM3 Combination equation (2011) is unlikely to accurately measure the EE by using wrist-mounted accelerometer-based physical activity sensors, for the values can be somewhat underestimated. This study reports that the vector magnitude parameters of accelerometer, body weight and HRR parameters can improve EE estimates, particularly those based on data from wrist-mounted accelerometer-based physical activity sensors. Although most 
307

308

309

310

311

312

313

314

315

316

317

318

319

320

321

322

323

324

325

326

327

328

329

330

331

332

333

334

335

336

337

338

339

340

341

342

343

344

345

346

347

348

349

350

351

of the commercial smart watches today have built-in accelerometer-based physical activity monitoring modules and optical heart rate sensors on the bottom, it is interesting to note that the data of these two devices are not integrated. Therefore, we suggest that the data of these two devices and our study results can be combined to develop a more accurate and reliable form of EE measurement technology.

\section{References}

ActiGraph. What is the difference among the energy expenditure algorithms? (Freedson VM3 Combination, 2011). Available at https://actigraphcorp.force.com/support/s/article/What-isthe-difference-among-the-Energy-Expenditure-Algorithms (Accessed 8 November 2018).

American College of Sports Medicine. Exercise is medicine. Available at https://www.exerciseismedicine.org/support_page.php/provider-action-guidel (accessed 28 May 2019).

American Heart Association. American Heart Association Recommendations for Physical Activity in Adults: American Heart Association. Available at https://www.heart.org/en/healthy-living/fitness/fitness-basics/aha-recs-for-physical-activityin-adults (accessed 28 May 2019).

Arieli R, \& Constantini N. 2012. Energy balance among female athletes. Harefuah 151(2):8285.

Beltrame T, Amelard R, Villar R, Shafiee MJ, Wong A, Hughson RL. 2016. Estimating oxygen uptake and energy expenditure during treadmill walking by neural network analysis of easy-to-obtain inputs. Journal of Applied Physiology (1985) 121(5):1226-1233 Doi: 10.1152/japplphysiol.00600.2016.

Bouchard DR, Trudeau F. 2008. Estimation of energy expenditure in a work environment: comparison of accelerometry and oxygen consumption/heart rate regression. Ergonomics 51(5):663-670 Doi: 10.1080/00140130701780484.

Brage S, Westgate K, Franks PW, Stegle O, Wright A, Ekelund U, Wareham NJ. 2015. Estimation of Free-Living Energy Expenditure by Heart Rate and Movement Sensing: A Doubly-Labelled Water Study. PLoS One 10(9):e0137206 Doi: 10.1371/journal.pone.0137206.

Burrows T, Harries SK, Williams RL, Lum C, Callister R. 2016. The Diet Quality of Competitive Adolescent Male Rugby Union Players with Energy Balance Estimated Using Different Physical Activity Coefficients. Nutrients 8(9):pii: E548 Doi: 10.3390/nu8090548.

Chang CH, Lin KC, Ho CS, Huang CC. 2019. Accuracy of the Energy Expenditure during Uphill Exercise Measured by the Waist-worn ActiGraph. Journal of Exercise Science \& Fitness 17(2):62-66 Doi: 10.1016/j.jesf.2019.01.003.

Chen X, Yao R, Yin G, Li J. 2017. Consecutive ultra-short-term heart rate variability to track dynamic changes in autonomic nervous system during and after exercise. Physiological Measurement 38(7):1384-1395 Doi: 10.1088/1361-6579/aa52b3.

Chomistek AK, Yuan C, Matthews CE, Troiano RP, Bowles HR, Rood J, Barnett JB, Willett WC, Rimm EB, Bassett DR Jr. 2017. Physical Activity Assessment with the ActiGraph GT3X and Doubly Labeled Water. Medicine \& Science in Sports \& Exercise 49(9):1935-1944 Doi: 10.1249/MSS.0000000000001299.

Colosio AL, Pedrinolla A, Da Lozzo G, Pogliaghi S. 2018. Heart Rate-Index Estimates Oxygen Uptake, Energy Expenditure and Aerobic Fitness in Rugby Players. Journal of Sports Science

Peer) reviewing PDF | (2019:06:38267:2:0:NEW 28 Sep 2019) 
352

353

354

355

356

357

358

359

360

361

362

363

364

365

366

367

368

369

370

371

372

373

374

375

376

377

378

379

380

381

382

383

384

385

386

387

388

389

390

391

392

393

394

395

396

397

and Medicine 17(4):633-639.

Crouter SE, Clowers KG, Bassett, DRJr. 2006. A novel method for using accelerometer data to predict energy expenditure. Journal of Applied Physiology (1985) 100(4):1324-1331 DOI: 10.1152/japplphysiol.00818.2005.

Delisle TT, Werch CE, Wong AH, Bian H, \& Weiler R. 2010. Relationship between frequency and intensity of physical activity and health behaviors of adolescents. Journal of School Health 80(3):134-140 Doi: 10.1111/j.1746-1561.2009.00477.x.

Domene PA, Easton C. 2014. Combined triaxial accelerometry and heart rate telemetry for the physiological characterization of Latin dance in non-professional adults. Journal of Dance Medicine \& Science 18(1):29-36 Doi: 10.12678/1089-313X.18.1.29.

Dong JG. 2016. The role of heart rate variability in sports physiology. Experimental and Therapeutic Medicine 11(5):1531-1536 Doi: 10.3892/etm.2016.3104.

Fisher JP, Young CN, Fadel PJ. 2015. Autonomic adjustments to exercise in humans. Comprehensive Physiology 5(2):475-512 Doi: 10.1002/cphy.c140022.

Gao Z, Wang X, Zhuo Q, Wang J, Hu F, Piao J, Liu F, Cao H. 2012. Energy expenditure on different physical activities of rural adults in North China. Journal of hygiene research 41(1):75-79.

Garber CE, Blissmer B, Deschenes MR, Franklin BA, Lamonte MJ, Lee IM, Nieman DC, Swain DP. 2011. American College of Sports Medicine position stand. Quantity and quality of exercise for developing and maintaining cardiorespiratory, musculoskeletal, and neuromotor fitness in apparently healthy adults: guidance for prescribing exercise. Medicine \& Science in Sports \& Exercise 43(7):1334-1359 Doi: 10.1249/MSS.0b013e318213fefb.

González K, Fuentes J, Márquez JL. 2017. Physical Inactivity, Sedentary Behavior and Chronic Diseases. Korean Journal of Family Medicine 38(3):111-115 Doi: 10.4082/kjfm.2017.38.3.111.

Hildebrand M, VAN Hees VT, Hansen BH, Ekelund U. 2014. Age group comparability of raw accelerometer output from wrist- and hip-worn monitors. Medicine \& Science in Sports \& Exercise 46(9):1816-1824 Doi: 10.1249/MSS.0000000000000289.

Kokkinos P, Myers J. 2010. Exercise and physical activity: clinical outcomes and applications. Circulation 122(16):1637-1648 Doi: 10.1161/CIRCULATIONAHA.110.948349.

Kuo TBJ, Li JY, Chen CY, Lin YC, Tsai MW, Lin SP, Yang CCH. 2018. Influence of Accelerometer Placement and/or Heart Rate on Energy Expenditure Prediction during Uphill Exercise. Journal of Motor Behavior 50(2):127-133 Doi: 10.1080/00222895.2017.1306481.

Lawrence MM, Cooley ID, Huet YM, Arthur ST, Howden R. 2014. Factors influencing isometric exercise training-induced reductions in resting blood pressure. Scandinavian Journal of Medicine \& Science in Sports 25(2):131-142 Doi: 10.1111/sms.12225.

Lee JM, Saint-Maurice PF, Kim Y, Gaesser GA, Welk G. 2016. Activity Energy Expenditure in Youth: Sex, Age, and Body Size Patterns. Journal of Physical Activity and Health 13(6 Suppl 1):S62-70 Doi: 10.1123/jpah.2016-0014.

Lyden K, Kozey SL, Staudenmeyer JW, Freedson PS. 2011. A comprehensive evaluation of commonly used accelerometer energy expenditure and MET prediction equations. European Journal of Applied Physiology 111(2):187-201 Doi: 10.1007/s00421-010-1639-8.

Menai M, van Hees VT, Elbaz A, Kivimaki M, Singh-Manoux A, Sabia S. 2017. Accelerometer assessed moderate-to-vigorous physical activity and successful ageing: results from the Whitehall II study. Scientific Reports 8:45772 Doi: 10.1038/srep45772.

Montoye AH, Mudd LM, Biswas S, Pfeiffer KA. 2015. Energy Expenditure Prediction Using

Peer) reviewing PDF | (2019:06:38267:2:0:NEW 28 Sep 2019) 
402

403

404

405

406

407

408

409

410

411

412

413

414

415

416

417

418

419

420

421

422

423

424

425

426

427

428

429

430

431

432

433

434

435

436

437

438

439

440

441

442

443

Raw Accelerometer Data in Simulated Free Living. Medicine \& Science in Sports \& Exercise 47(8):1735-1746 Doi: 10.1249/MSS.0000000000000597.

Oppert JM, Charles MA, Charreire H, Menai M, De Bourdeaudhuij I, Brage S, de LauzonGuillain B, Fagherazzi G, Balkau B. 2016. Home and Work Physical Activity Environments: Associations with Cardiorespiratory Fitness and Physical Activity Level in French Women. International Journal of Environmental Research and Public Health 13(8):pii: E824 Doi: 10.3390/ijerph13080824.

Rousset S, Fardet A, Lacomme P, Normand S, Montaurier C, Boirie Y, Morio B. 2015. Comparison of total energy expenditure assessed by two devices in controlled and free-living conditions. European Journal of Sport Science 15(5):391-399 Doi: 10.1080/17461391.2014.949309.

Schneller MB, Pedersen MT, Gupta N, Aadahl M, Holtermann A. 2015. Validation of five minimally obstructive methods to estimate physical activity energy expenditure in young adults in semi-standardized settings. Sensors (Basel) 15(3):6133-6151 Doi: 10.3390/s150306133.

Shih Y, Ho CS, Shiang TY. 2014. Measuring kinematic changes of the foot using a gyro sensor during intense running. Journal of Sports Sciences 32(6):550-556 Doi: 10.1080/02640414.2013.843013.

Sirichana W, Dolezal BA, Neufeld EV, Wang X, Cooper CB. 2017. Wrist-worn triaxial accelerometry predicts the energy expenditure of non-vigorous daily physical activities. Journal of Science and Medicine in Sport 20(8):761-765 Doi: 10.1016/j.jsams.2017.01.233.

Strath SJ, Bassett DR Jr, Swartz AM, Thompson DL. 2001. Simultaneous heart rate-motion sensor technique to estimate energy expenditure. Medicine \& Science in Sports \& Exercise 33(12):2118-2123.

Tarp J, Andersen LB, Østergaard L. 2015. Quantification of Underestimation of Physical Activity During Cycling to School When Using Accelerometry. Journal of Physical Activity and Health 12(5):701-707. Doi: 10.1123/jpah.2013-0212.

Tudor-Locke C, Barreira TV, Schuna JMJr. 2015. Comparison of step outputs for waist and wrist accelerometer attachment sites. Medicine \& Science in Sports \& Exercise 47(4):839-842 Doi: 10.1249/MSS.0000000000000476.

Villars C, Bergouignan A, Dugas J, Antoun E, Schoeller DA, Roth H, Maingon AC, Lefai E, Blanc S, Simon C. 2012. Validity of combining heart rate and uniaxial acceleration to measure free-living physical activity energy expenditure in young men. Journal of Applied Physiology (1985) 113(11):1763-1771 Doi: 10.1152/japplphysiol.01413.2011.

Weir JB. 1949. New methods for calculating metabolic rate with special reference to protein metabolism. The Journal of Physiology 109(1-2):1-9.

Welch WA, Bassett DR, Thompson DL, Freedson PS, Staudenmayer JW, John D, Steeves JA, Conger SA, Ceaser T, Howe CA, Sasaki JE, Fitzhugh EC. 2013. Classification accuracy of the wrist-worn gravity estimator of normal everyday activity accelerometer. Medicine \& Science in Sports \& Exercise 45(10):2012-2019 Doi: 10.1249/MSS.0b013e3182965249.

Westerterp KR. 2017. Doubly labelled water assessment of energy expenditure: principle, practice, and promise. European Journal of Applied Physiology 117(7):1277-1285 Doi: 10.1007/s00421-017-3641-x.

White T, Westgate K, Wareham NJ, Brage S. 2016. Estimation of Physical Activity Energy Expenditure during Free-Living from Wrist Accelerometry in UK Adults. PLoS One

Peer) reviewing PDF | (2019:06:38267:2:0:NEW 28 Sep 2019) 
444

445

446

447

448

449

450

451

452

453

454

455

456 11(12):e0167472 Doi: 10.1371/journal.pone.0167472.

Poobalan A, Aucott L. 2016. Obesity Among Young Adults in Developing Countries: A Systematic Overview. Current Obesity Reports 5(1):2-13. Doi: 10.1007/s13679-016-0187-x.

Xu C, Quan M, Zhang H, Zhou C, Chen P. 2018. Impact of parents' physical activity on preschool children's physical activity: a cross-sectional study. PeerJ 6:e4405 Doi: 10.7717/peerj.4405.

Yang Y, Schumann M, Le S, Cheng S. 2018. Reliability and validity of a new accelerometerbased device for detecting physical activities and energy expenditure. PeerJ 6:e5775 Doi: 10.7717/peerj.5775.

Żukiewicz-Sobczak W, Wróblewska P, Zwoliński J, Chmielewska-Badora J, Adamczuk P, Krasowska E, Zagórski J, Oniszczuk A, Piątek J, Silny W. 2014. Obesity and poverty paradox in developed countries. Annals of Agricultural and Environmental Medicine 21(3):590-594. Doi: 10.5604/12321966.1120608. 
Table $\mathbf{1}$ (on next page)

Anthropometric characteristics of participants. 


\section{Table 1:}

2 Anthropometric characteristics of participants.

\begin{tabular}{cccccc}
\hline & Age $(\mathrm{yrs})$ & Height $(\mathrm{cm})$ & BMI $\left(\mathrm{kg} / \mathrm{m}^{2}\right)$ & Body weight $(\mathrm{kg})$ & Sex \\
\hline Mean & 22.90 & 168.05 & 22.52 & 63.90 & 49 males, 41 \\
SD & 4.15 & 7.62 & 3.25 & 12.06 & females \\
\hline
\end{tabular}

3

4 


\section{Table 2 (on next page)}

Comparison of measured EE by Vmax (CMEE) and estimated EE by GT9X-EE in 5 treadmill walking/running tests (mean \pm SD). 


\section{Table 2:}

2 Comparison of measured EE by Vmax (CMEE) and estimated EE by GT9X-EE in 5 treadmill

3 walking/running tests (mean \pm SD).

\begin{tabular}{|c|c|c|c|c|c|c|c|}
\hline Position & $\begin{array}{l}\text { Treadmill Speed } \\
\qquad(\mathrm{km} / \mathrm{h})\end{array}$ & $\begin{array}{c}\mathrm{CMEE} \\
\left(\mathrm{kcal} \cdot \mathrm{kgw}^{-1} \cdot \mathrm{min}^{-1}\right)\end{array}$ & 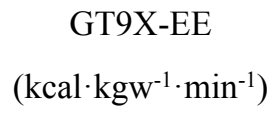 & ES & $\begin{array}{l}\text { MAPE } \\
(\%)\end{array}$ & $\mathrm{r}$ & ICC \\
\hline \multirow[t]{5}{*}{ Wrist } & 4.80 & $0.070 \pm 0.009$ & $0.055 \pm 0.010^{*}$ & 1.58 & 21.43 & 0.105 & \multirow{5}{*}{0.073} \\
\hline & 6.42 & $0.111 \pm 0.014$ & $0.065 \pm 0.008^{*}$ & 4.03 & 41.44 & 0.030 & \\
\hline & 8.04 & $0.148 \pm 0.010$ & $0.071 \pm 0.005^{*}$ & 9.74 & 52.03 & -0.169 & \\
\hline & 9.66 & $0.172 \pm 0.012$ & $0.072 \pm 0.005^{*}$ & 10.88 & 58.14 & -0.165 & \\
\hline & 11.28 & $0.202 \pm 0.015$ & $0.073 \pm 0.005^{*}$ & 11.54 & 63.86 & -0.130 & \\
\hline \multirow[t]{5}{*}{ Hip } & 4.80 & $0.070 \pm 0.009$ & $0.066 \pm 0.013$ & 0.36 & 5.71 & $0.529 * *$ & \multirow{5}{*}{0.868} \\
\hline & 6.42 & $0.111 \pm 0.014$ & $0.104 \pm 0.021 *$ & 0.39 & 6.31 & $0.428^{* *}$ & \\
\hline & 8.04 & $0.148 \pm 0.010$ & $0.141 \pm 0.026^{*}$ & 0.36 & 4.73 & 0.110 & \\
\hline & 9.66 & $0.172 \pm 0.012$ & $0.163 \pm 0.023 *$ & 0.49 & 5.23 & $0.210^{* *}$ & \\
\hline & 11.28 & $0.202 \pm 0.015$ & $0.181 \pm 0.023^{*}$ & 1.08 & 10.40 & 0.162 & \\
\hline
\end{tabular}

\section{Notes.}

$5 *$ Significantly different from CMEE, $p<0.05$.

$6 * *$ Significant correlation with CMEE, $p<0.001$.

7 Mean values \pm standard deviation (SD); CMEE, criterion measure energy expenditure; GT9X, ActiGraph GT9X-Link

8 accelerometer; ES, Effect size (Cohen's d); Mean Absolute Percentage Error (MAPE) $=\{[\mid$ (Predicted value - Actual

9 value) $\mid$ /Actual value $\left.{ }^{*} 100\right\} / \mathrm{n}$; r, Pearson coefficient of determination; ICC, intraclass correlation coefficient. 


\section{Table 3 (on next page)}

Modified models to predict EE $\left(\mathrm{kcal}^{\mathrm{kggw}}{ }^{-1} \cdot \mathrm{min}^{-1}\right)$ from VM, BW, and HRR. 


\section{Table 3:}

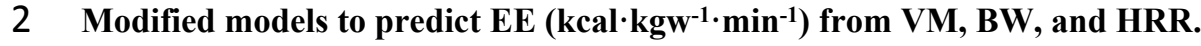

\begin{tabular}{clcc}
\hline Position & Prediction equation & $\mathrm{R}^{2}$ & $\mathrm{SEE}$ \\
\hline Wrist & $\mathrm{EE}=0.000003 \mathrm{VM}-0.000461 \mathrm{BW}+0.000585 \mathrm{HRR}+0.078066$ & .802 & 0.021 \\
Hip & $\mathrm{EE}=0.000009 \mathrm{VM}-0.000299 \mathrm{BW}+0.000682 \mathrm{HRR}+0.046825$ & .805 & 0.021 \\
\hline
\end{tabular}

\section{Notes.}

4 VM, vector magnitudes; BW, body weight in kgw; HRR, heart rate reserve; ${ }^{2}$, coefficient of determination; $S E E$, 5 standard error of estimate. 


\section{Table 4 (on next page)}

Validity and reliability analysis of traditional prediction equation and modified prediction equations. 


\section{Table 4:}

2 Validity and reliability analysis of traditional prediction equation and modified prediction equations.

\begin{tabular}{ccccc} 
& \multicolumn{2}{c}{ Freedson's VM3 Combination } & \multicolumn{2}{c}{ Modified models } \\
\hline Position & $\mathrm{r}$ & ICC & $\mathrm{r}$ & ICC \\
\hline Wrist & 0.620 & 0.073 & 0.895 & 0.863 \\
Hip & 0.885 & 0.868 & 0.897 & 0.889 \\
\hline
\end{tabular}

3 Notes.

4 r, Pearson coefficient of determination; ICC, intraclass correlation coefficient.

5

6 